\title{
Analysis of grape fruits and grape seed for their major, minor and trace elemental contact by XRF technique
}

\author{
Khalid H. Mahdi* Rafe'e K. Al-Kubaisy* Shakier M. Al-Jubory** \\ Rewidaa S. Hassan ***
}

Received 3, January, 2011

Accepted 18, July, 2011

\begin{abstract}
:
Grapes and grape seeds are important samples employed for environmental medical studies. The air of this work was to identify and concentration calculation of the elements in grapes fruit and thier seeds by using X-Ray fluoresces technique (XRF) . Samples were collected from Abo Ghraib of Baghdad city ,the grape seeds were obtained from those samples. Both samples were taken under experimental procedure to obtain the sample which were ready for analysis .

The samples were then submitted to experimental conditions using a radiation source and then samples were applied for counting analysis shows the elements $\mathrm{Na}$, $\mathrm{Mg}, \mathrm{Al}, \mathrm{Si}, \mathrm{P}, \mathrm{S}, \mathrm{Cl}, \mathrm{K}, \mathrm{Ca}$, and $\mathrm{Sr}$ as major components of the samples. $\mathrm{Fe}, \mathrm{Sr}$, $\mathrm{I}, \mathrm{Ba}$ and $\mathrm{V}$ were found an minor elements other elements $\mathrm{Cr}, \mathrm{Cu}, \mathrm{Sn}, \mathrm{Sb}, \mathrm{Te}$ gave a value of 5-10 ppm, $\mathrm{Co}, \mathrm{Ni}, \mathrm{Rb}, \mathrm{Ag}, \mathrm{Cd}<5 \mathrm{ppm}$ for the samples of grape seeds .

The analysis of grape seeds present $\mathrm{Sr}, \mathrm{Ca}, \mathrm{K}, \mathrm{Cl}, \mathrm{S}, \mathrm{Si}, \mathrm{P}$ as major components of the samples, and gave a values for $\mathrm{Cr}$ and $\mathrm{Rb}, \mathrm{Sn}, \mathrm{Sb}$ and $\mathrm{Te}(5-10$ ppm), while $\mathrm{Co}, \mathrm{Rb}, \mathrm{Ni}, \mathrm{Ag}, \mathrm{Cd}$ (Less than 5 ppm) .
\end{abstract}

Key words: XRF technique, grape seed.

\section{Introduction:}

It is evident that physicians of unani medicine were well a ware with the existence and importance of elements and its significant role in human health[1] Trace elements are crucial to virtually all biochemical and physiological process . they are present in tissues only in minute quantity micrograms to micrograms per grams of weight tissue of their iron , chlorate, iodine , copper, zinc, manganese, molybdenum, selenium, chromium, fluorine, silicon, strontium , cadmium, nickel, tin and vanadium are now thought to be essential for animal life. The signs of deficiency may be range in degree from an important of biochemical function to death and essential trace element may or not be practically important to human nutrition. Grapes is compared to milk in nutrition elements but even there are some proportion which is not present in milk .It is one of the rich fruits house good in body building . Some scientist regard it as complementary groups to enzymes.

Varity experimented models have shown that nutritional deficiencies or imbalance of trace elements such as chrome, iron, vanadium, copper and zinc results in elevation of serum levels of cholesterol[2]. XRF technique is one of the methods employed to

*College of Education/University of Baghdad

**College of Madenat Al-Alim/Ministry of Higher Education and Scientific Research

****Ministry of Sciences and Technology 
determine the elementals in biological samples .

This method is multi elemental method, nondestructive and cheap comparing with other methods.XRF has been used for the determination in our laboratory. concentration of $\mathrm{Na}$, $\mathrm{Mg}, \mathrm{Al}, \mathrm{K}, \mathrm{Si}, \mathrm{Ca}, \mathrm{Mn}, \mathrm{Fe}, \mathrm{S}$, and $\mathrm{P}$ as major elements $\mathrm{Ti}, \mathrm{Cl}, \mathrm{Rh}$ and $\mathrm{Sr}$ as minor elements and $\mathrm{Cu}, \mathrm{Zn}, \mathrm{V}, \mathrm{Cr}, \mathrm{Ca}$, as trace elements in the tea samples collected from markets [3] .The group seed servers cultivar is(Aluminum prance, cuircles muscle or harburg Althouse analysed by nortein oil, oil tare ,usfall composition and element content. All results at micro and macro amounts of the elements [4] Major and Trace elements $\mathrm{K}, \mathrm{Ca}, \mathrm{Mg}, \mathrm{P}, \mathrm{Na}$, as major $\mathrm{Br}, \mathrm{Sr}$, $\mathrm{Mo}, \mathrm{Fe}, \mathrm{Cu}, \mathrm{Ni}, \mathrm{Ba}, \mathrm{Mn}$, $\mathrm{Zn}, \mathrm{Rb}, \mathrm{Sn}$ as ultra trace elements were more determent using XRF technique[5] .

To complete the total Iraqi food angle programe which has been started since 1987 Cd- 109 a mention of radioisotopes sources. The elements concentration of $\mathrm{K}, \mathrm{Fe}, \mathrm{Cu}, \mathrm{Zn}$, and $\mathrm{Sr}$ miler determined using XRF system . Results show that high concentration of $\mathrm{K}, \mathrm{Fe}, \mathrm{Sr}$ and $\mathrm{Ge}$ concentration beet and celery affer from location to another . [6]

Radioisotopes Cd-109 and Fe-55 has been used for the determination of $\mathrm{Ca}, \mathrm{Na}, \mathrm{Mg}, \mathrm{Cl}, \mathrm{K}$ in lraqi's date samples [7] . Our investigative consistent collecting different origion grape and grape seeds and analysis of these samples using the well established methods. Then contact element were determent using XRF technique and the results were disused

$\mathrm{XRF}$ is one of the powerful technique for angles of trace elements in biological samples and other materials especially for low $\mathrm{Z}$ number elements . XRF generated by bombarding matter with high- energy particles such as electrons or alpha particles or X-ray photons . when an atom is go bombarded, an electron is ejected from one of inner shells of the atom this vacancy is immediately filled by an electron from a higher energy shell, creating a vacancy in that shell that is, in turn, filled by an electron from a yet higher shell.

$\mathrm{X}$ - ray technique has been employed for the analysis of Iraqi vegetables such as okra, carrot, cabbadge ....etc. [8] . Qeadhuns has determined the trace elements in different types of vegetable using XRF technique [9] .It was very high value of $\mathrm{K}, \mathrm{Ca}, \mathrm{P}, \mathrm{Mg}, \mathrm{Fe}, \mathrm{Na}$ and $\mathrm{Mn}$ from sugar and oil grape is one of the fruits that regicidal as one of this type of fruit [7]. It is important for near discares are central Narre system [10].

It has been used in disorders liver and spleen clear to the presence of suger in it which grape is regarded as fast food as having nutrition value and used to prevent kidney problem it is also content of compound which is resemble to insulin therefore it is always advanced to here a special of grape which is high content to organic and inorganically the green grape is used to treat the thought and in rejecting headache.

\section{Materrial and Methods: Sample preparatren}

Grape and grape seeds has been collected from Abo Ghraib of Baghdad City. The samples were kept in the oven (vector types ) at $60{ }^{\circ} \mathrm{C}$ for 2 hours and drying . the dried samples were graned to fine hours genius power using an electrical agate mortor. The samples were prepared in pellets of a diameter of $32 \mathrm{~mm}$.

\section{Analysis}

A Germany system was used for the analysis containing $\mathrm{Si}(\mathrm{Li})$ flash type detector which does not 
need liquid nitrogen for cooling ,The system can be operated at $25{ }^{\circ} \mathrm{C}$ it has a source as tube from radium window using three targets $(\mathrm{Pd}, \mathrm{Ti}, \mathrm{W})$ to provide X-ray with different energies in order to cover the large range of spectrum using vacuum system with pressure $10 \mathrm{~mm} \mathrm{Hg}$ helped in getting more efficiency of analysis especially for higher elements the total exposure time is $900 \mathrm{sec}$ for three targets (300 sec for each target). Then all information about the data analysis or spectrum of the samples are driven from a computer that added to the system .

\section{Results and Discussions:}

Eleven samples of grapes and their seeds were collected and treated according to prouder recommended by our laboratory for the perpose of getting a role samples ready for analysis table.(1).

Table -2- gives the concentration of elements $\mathrm{Na}, \mathrm{Mg}, \mathrm{Al}, \mathrm{Si}, \mathrm{P}, \mathrm{S}$, $\mathrm{Cl}, \mathrm{K}, \mathrm{Ca}$ and $\mathrm{Zr}$ as major elements . Table -3- shows the minor elements $\mathrm{Fe}, \mathrm{Sr}, \mathrm{Mo}, \mathrm{I}, \mathrm{Ba}$ and $\mathrm{V}$ in grape fruits samples, and the detection limite of each elements .

Table -4- gives the concentration of $\mathrm{Ti}, \mathrm{Cr}, \mathrm{Mn}, \mathrm{Co}, \mathrm{Ni}, \mathrm{Cu}, \mathrm{Zn}, \mathrm{Br}$ and $\mathrm{Rb}$ as a trac elements . Table -5gives us the concentration of major elements $\mathrm{Na}, \mathrm{Mg}, \mathrm{Si}, \mathrm{P}, \mathrm{S}, \mathrm{Cl}, \mathrm{K}$, $\mathrm{Ca}$ and $\mathrm{Sr}$ in the grap seeds. While table -6- present the concentration of $\mathrm{Al}, \mathrm{V}, \mathrm{Cr}, \mathrm{Zn}, \mathrm{Fe}, \mathrm{Mo}, \mathrm{I}, \mathrm{Ba}, \mathrm{Mn}$, and $\mathrm{Sr}$ as minor elements detected in grap seeds . Table -7- present trac and altratrace elements detected in the grap seeds, it gives the concentration of $\mathrm{Cr}, \mathrm{Co}, \mathrm{Ni}, \mathrm{Cu}, \mathrm{Pb}, \mathrm{Ag}, \mathrm{Cd}, \mathrm{Sn}$, $\mathrm{Sb}, \mathrm{W}, \mathrm{Te}, \mathrm{V}$ and ultratrace elements $\mathrm{Br}, \mathrm{Pb}$, and $\mathrm{Th}$ in these grap seeds .

Major elements deformed for grape samples show values for Na only in the sample ZK.1 (table-2)which give a value of $0.205 \%$ may give a concentrate value of $\mathrm{Cl}, \mathrm{S}, \mathrm{P}, \mathrm{Si}$ and $\mathrm{Al}$ all gave a very low value all between $0.01-0.154 \%$ Potassium give a concentration value between 0.717 $1.608 \%$ of all elements, the minor elements $\mathrm{Fe}, \mathrm{Sr}, \mathrm{Mo}, 1, \mathrm{Ba}$ and $\mathrm{V}$ gave a concentration value less than $1 \mathrm{mg} / \mathrm{g}$ in all the analyzed samples except at ZK-2, which gave a value of $1.246 \mathrm{mg} / \mathrm{g}$. For trace elements which were investigated using the same method of analysis chromic show a value of 3.7-9.9 $\mathrm{ppm}$ or $(\mu \mathrm{g} / \mathrm{g})$ which for in 4.8-6.9 ppm it was reported to be $\mathrm{Cu}$ between 2.9-8.9 ppm this a good indicator for guardians Co were arranged to (0.6-2.0) ppm , $\operatorname{Br}(0.2$ 1.2) $\mathrm{ppm}$ while $\mathrm{Zn}$ gave account rater range 1.9-15.4 ppm. The above returned results showed that grape are not highly concentration with the elements present in the field where they grown(tables 2-4).

One can see (table -5) regarding samples grape seeds of the elements determented $\mathrm{Na}, \mathrm{Mg}, \mathrm{Si}, \mathrm{P}, \mathrm{S}, \mathrm{Cl}$, $\mathrm{K}, \mathrm{Ca}, \mathrm{Sr}$, all these elements were in the concentration values are higher for $\mathrm{Mg}, \quad \mathrm{P}, \quad \mathrm{S}, \mathrm{K}$ and $\mathrm{Ca}$ which concentration values for the $\mathrm{Na}, \mathrm{Si}, \mathrm{Cl}$ ,Sr are found to be lower than the above mentioned . the minor elements $\mathrm{Al}, \mathrm{V}, \mathrm{Cr}, \mathrm{Zn}, \mathrm{Fe}, \mathrm{Mo}, \mathrm{I}, \mathrm{Ba}$,Mn, and $\mathrm{Sr}$ gave a concentration value less than $1 \mathrm{mg} / \mathrm{g}$ in all samples(table-6).

Trace elements in the seed of the grape chromic gave a value of 16.4 for sample $\mathrm{SZK}-7, \mathrm{Rb}$ value give a value of $10.9 \mathrm{ppm}$ for sample SZK-8. For the other elements $\mathrm{Cu}, \mathrm{Sn}, \mathrm{Sb}, \mathrm{Te}$, give values higher than the range 5-10 ppm, while the other elements $\mathrm{Co}, \mathrm{Ni}$ , $\mathrm{Rb}, \mathrm{Ag}, \mathrm{Cd}$, gave less than 5 ppm , Te give a value of $8.2-5 \mathrm{ppm} . \mathrm{Br}$, $\mathrm{Pb}$ and $\mathrm{Th}$ where found at concentration lower than elements determined the concentration values range 0.5 - 3 ppm (table-7).

This methode improved the ability of analysis for multi-elements 
determination of elements in the sample, which is giving a dictionary values for the content grapes and their seed. Grapes is well known as a medicine for certain deseases .

Table (1) Signs employed for the samples recognization

\begin{tabular}{|l|l|l|}
\hline Samples & Grapes Symbols & seeds \\
\hline Kamali & ZK-1 & SZK-1 \\
\hline Rishmo & ZK-2 & SZK-2 \\
\hline Shadasoda & ZK-3 & SZK-3 \\
\hline Dise Al nize & ZK-4 & SZK-4 \\
\hline Abassi & ZK-5 & SZK-5 \\
\hline Shada bayda & ZK-6 & SZK-6 \\
\hline Halawani & ZK-7 & SZK-7 \\
\hline Romi Aswad & ZK-8 & SZK-8 \\
\hline Baitamoni & ZK-9 & SZK-9 \\
\hline Aswad Aynoni & ZK-10 & SZK-10 \\
\hline King Romi & ZK-11 & SZK-11 \\
\hline
\end{tabular}

Table(2) Major concentration for all types of grapes \%

\begin{tabular}{|c|c|c|c|c|c|c|c|c|c|c|c|c|}
\hline Element & ZK-1 \% & $\begin{array}{c}\mathrm{Zk}-2 \\
\%\end{array}$ & $\begin{array}{c}\mathrm{ZK}-3 \\
\%\end{array}$ & $\begin{array}{c}\text { ZK-4 } \\
\%\end{array}$ & $\begin{array}{c}\text { ZK-5 } \\
\%\end{array}$ & $\begin{array}{c}\text { ZK-6 } \\
\%\end{array}$ & $\begin{array}{c}\text { ZK-7 } \\
\%\end{array}$ & $\begin{array}{c}\text { ZK- } 8 \\
\%\end{array}$ & $\begin{array}{c}\text { ZK-9 } \\
\%\end{array}$ & $\begin{array}{c}\mathrm{ZK}-10 \\
\%\end{array}$ & $\begin{array}{c}\text { ZK-11 } \\
\%\end{array}$ & Detection Limit \\
\hline $\mathrm{Na}$ & 0.205 & $<0.087$ & $<0.071$ & 0.0136 & $<0.048$ & 0.073 & $<0.071$ & $<0.077$ & $<0.120$ & $<0.086$ & 0.090 & 0.01466 \\
\hline $\mathrm{Mg}$ & 0.072 & 0.0101 & 0.084 & 0.069 & 0.035 & 0.071 & 0.034 & 0.096 & 0.139 & 0.074 & 0.078 & 0.06 \\
\hline $\mathrm{Al}$ & 0.027 & 0.171 & 0.050 & 0.026 & 0.010 & 0.030 & 0.192 & 0.035 & 0.052 & 0.0196 & 0.038 & 0.0317 \\
\hline $\mathrm{Si}$ & 0.097 & 0.187 & 0.137 & 0.101 & 0.042 & 0.0101 & 0.072 & 0.133 & 0.145 & 0.090 & 0.116 & 0.014 \\
\hline $\mathrm{P}$ & 0.167 & 0.135 & 0.102 & 0.167 & 0.095 & 0.115 & 0.146 & 0.149 & 0.154 & 0.164 & 0.122 & 0.00514 \\
\hline$S$ & 0.099 & 0.108 & 0.068 & 0.094 & 0.039 & 0.068 & 0.073 & 0.106 & 0.209 & 0.084 & 0.066 & 0.001 \\
\hline $\mathrm{Cl}$ & 0.095 & 0.084 & 0.059 & 0.863 & 0.038 & 0.034 & 0.037 & 0.036 & 0.107 & 0.089 & 0.049 & 0.001 \\
\hline $\mathrm{K}$ & 1.212 & 0.0918 & 0.904 & 1.213 & 0.717 & 0.836 & 1.196 & 1.652 & 1.608 & 1.232 & 0.965 & 0.0124 \\
\hline $\mathrm{Ca}$ & 0.127 & 0.128 & 0.110 & 0.111 & 0.038 & 0.073 & 0.0107 & 0.138 & 0.070 & 0.111 & 0.113 & 0.0057 \\
\hline $\mathrm{Zr}$ & bdL & bdL & bdL & bdL & $<0.05$ & $<0.05$ & $<0.05$ & bdL & $<0.05$ & bdL & 0.002 & 0.00008 \\
\hline
\end{tabular}

Table (3) Minor elements concentration for all types of grapes(mg/g)

\begin{tabular}{|c|c|c|c|c|c|c|c|c|c|c|c|c|}
\hline Element & ZK-1 & ZK-2 & ZK-3 & ZK-4 & ZK-5 & ZK-6 & ZK-7 & ZK- 8 & ZK-9 & ZK-10 & ZK-11 & Detection Limit \\
\hline $\mathrm{Fe}$ & 0.084 & 1.246 & 0.0131 & 0.067 & 0.054 & 0.075 & 0.052 & 0.078 & 0.093 & 0.057 & 0.069 & 0.0011 \\
\hline $\mathrm{Sr}$ & 0.020 & 0.017 & 0.015 & 0.021 & 0.010 & 0.013 & 0.015 & 0.014 & 0.018 & 0.015 & 0.016 & 0.00005 \\
\hline $\mathrm{Mo}$ & 0.015 & 0.014 & $<0.014$ & $<0.014$ & $<0.017$ & $<0.021$ & $<0.016$ & $<0.013$ & $<0.02$ & $<0.002$ & $<0.014$ & 0.0002 \\
\hline $\mathrm{I}$ & 0.024 & 0.019 & 0.025 & 0.023 & 0.036 & 0.047 & 0.021 & $<0.013$ & 0.023 & $<0.017$ & $<0.018$ & 0.0003 \\
\hline $\mathrm{Ba}$ & 0.032 & 0.061 & 0.025 & 0.029 & $<0.045$ & $<0.039$ & 0.048 & 0.040 & $<0.034$ & $<0.034$ & $<0.035$ & 0.00025 \\
\hline $\mathrm{V}$ & 0.012 & 0.011 & 0.008 & 0.010 & $<0.005$ & $<0.005$ & 0.010 & 0.007 & 0.015 & 0.010 & 0.007 & 0.001 \\
\hline
\end{tabular}


Table (4) Trace elements concentration for all types of grapes $(\mu \mathrm{g} / \mathrm{g}) \mathrm{or}(\mathrm{ppm})$

\begin{tabular}{|c|c|c|c|c|c|c|c|c|c|c|c|c|}
\hline Element & ZK-1 & ZK-2 & ZK-3 & ZK-4 & ZK-5 & ZK-6 & ZK-7 & ZK-8 & ZK-9 & ZK-10 & ZK-11 & Detection Limit \\
\hline $\mathrm{Ti}$ & $<5.0$ & $<5.0$ & $<5.0$ & $<5.0$ & $<5.0$ & $<5.0$ & $<5.0$ & $<5.0$ & $<5.0$ & $<5.0$ & $<5.0$ & 0.00015 \\
\hline $\mathrm{Cr}$ & 4.9 & $<5.0$ & 9.9 & 5.0 & 4.2 & 3.7 & $<4.6$ & $<5.4$ & 5.5 & $<4.9$ & $<4.3$ & 0.0002 \\
\hline $\mathrm{Mn}$ & 4.8 & 7.3 & 6.4 & 6.9 & 3.3 & 5.6 & 4.0 & 5.7 & 4.2 & 6.4 & 7.1 & 0.00012 \\
\hline $\mathrm{CO}$ & 0.6 & 1.3 & 1.0 & 1.2 & 0.8 & 0.9 & 1.1 & 1.3 & 1.4 & 2.0 & 1.0 & 0.001 \\
\hline $\mathrm{Ni}$ & 0.6 & 3.0 & 1.5 & 0.7 & 0.4 & $<0.7$ & $<0.8$ & 1.1 & 12.1 & 0.6 & 0.8 & 0.0001 \\
\hline $\mathrm{Cu}$ & 5.0 & 6.0 & 4.8 & 5.1 & 2.9 & 4.3 & 3.4 & 8.9 & 4.0 & 4.1 & 3.3 & 0.0002 \\
\hline $\mathrm{Zn}$ & 6.5 & 15.4 & 8.2 & 5.8 & 2.6 & 3.2 & 3.2 & 2.9 & 3.4 & 1.9 & 2.8 & 0.00098 \\
\hline $\mathrm{Br}$ & 1.2 & 0.9 & 0.6 & 1.2 & 0.0 & 1.0 & 1.0 & 0.2 & 0.7 & 0.9 & 0.9 & 0.00006 \\
\hline $\mathrm{Rb}$ & 4.4 & 2.2 & 3.6 & 1.9 & 2.9 & $<7.0$ & 3.1 & 29.8 & 3.8 & 2.5 & $<3.0$ & 0.00005 \\
\hline $\mathrm{Sn}$ & $<4.2$ & $<3.7$ & $<3.7$ & $<4.8$ & $<5.3$ & $<3.7$ & $<4.0$ & $<3.5$ & $<0.7$ & $<5.1$ & $<5.2$ & 0.00001 \\
\hline $\mathrm{Sb}$ & $<6.2$ & $<5.6$ & $<5.2$ & $<6.1$ & $<8.0$ & $<8.0$ & $<4.4$ & $<4.8$ & $<9.0$ & $<6.1$ & $<7.3$ & 0.00008 \\
\hline $\mathrm{Te}$ & $<9.5$ & 15.9 & 6.9 & $<10$ & $<11$ & $\mathrm{bdL}$ & $\mathrm{bdL}$ & $\mathrm{bdL}$ & $\mathrm{bdL}$ & $\mathrm{bdL}$ & $\mathrm{bdL}$ & 0.0001 \\
\hline
\end{tabular}

Table (5) Major elements concentration for all types grape seeds

\begin{tabular}{|c|c|c|c|c|c|c|c|c|c|c|c|}
\hline Element & $\begin{array}{c}\text { SZK-1 } \\
\%\end{array}$ & $\begin{array}{c}\text { SZK-2 } \\
\%\end{array}$ & $\begin{array}{c}\text { SZK-3 } \\
\%\end{array}$ & $\begin{array}{c}\text { SZK-4 } \\
\%\end{array}$ & $\begin{array}{c}\text { SZK-5 } \\
\%\end{array}$ & $\begin{array}{c}\text { SZK-6 } \\
\%\end{array}$ & $\begin{array}{c}\text { SZK-7 } \\
\%\end{array}$ & $\begin{array}{c}\text { SZK-8 } \\
\%\end{array}$ & $\begin{array}{c}\text { SZK-9 } \\
\%\end{array}$ & $\begin{array}{c}\text { SZK-10 } \\
\%\end{array}$ & $\begin{array}{c}\text { SZK-11 } \\
\%\end{array}$ \\
\hline $\mathrm{Na}$ & $\mathrm{O} .091$ & 0.083 & 0.090 & 0.086 & 0.092 & 0.079 & 0.082 & 0.097 & 0.086 & 0.097 & 0.10 \\
\hline $\mathrm{Mg}$ & 0.235 & 0.253 & 0.196 & 0.268 & 0.232 & 0.239 & 0.246 & 0.247 & 0.194 & 0.2 & 0.193 \\
\hline $\mathrm{Si}$ & 0.019 & 0.031 & 0.016 & 0.016 & 0.0081 & 0.021 & 0.007 & 0.007 & 0.017 & 0.002 & 0.067 \\
\hline $\mathrm{P}$ & 0.381 & 0.295 & 0.361 & 0.323 & 0.424 & 0.325 & 0.310 & 0.416 & 0.286 & 0.475 & 0.377 \\
\hline $\mathrm{S}$ & 0.230 & 0.211 & 0.262 & 0.181 & 0.201 & 0.185 & 0.190 & 0.266 & 0.179 & 0.257 & 0.246 \\
\hline $\mathrm{Cl}$ & 0.032 & 0.020 & 0.024 & 0.030 & 0.020 & 0.016 & 0.016 & 0.019 & 0.025 & 0.023 & 0.020 \\
\hline $\mathrm{K}$ & 0.646 & 0.244 & 0.490 & 0.599 & 0.630 & 0.486 & 0.478 & 0.637 & 0.412 & 0.557 & 0.361 \\
\hline $\mathrm{Ca}$ & 0.875 & 1.08 & 0.965 & 0.906 & 0.764 & 0.802 & 0.764 & 0.637 & 0.977 & 0.923 & 1.308 \\
\hline $\mathrm{Sr}$ & 0.01 & 0.012 & 0.012 & 0.0111 & 0.008 & 0.010 & 0.010 & 0.010 & 0.010 & 0.010 & 0.014 \\
\hline
\end{tabular}

Table (6) Minor elements concentration for all tayps seeds of grapes(mg/g)

\begin{tabular}{|c|c|c|c|c|c|c|c|c|c|c|c|}
\hline Element & SZK-1 & SZK-2 & SZK-3 & SZK-4 & SZK-5 & SZK- & SZK-7 & SZK- & SZK-9 & SZK-10 & SZK-11 \\
\hline $\mathrm{Al}$ & $\mathrm{O} .030$ & 0.230 & 0.167 & 0.082 & 0.032 & 0.030 & 0.028 & 0.060 & 0.087 & 0.035 & 0.288 \\
\hline $\mathrm{V}$ & 0.010 & 0.013 & 0.005 & 0.007 & 0.008 & 0.010 & 0.008 & 0.015 & 0.015 & 0.007 & 0.014 \\
\hline $\mathrm{Zn}$ & 0.012 & 0.012 & 0.069 & 0.011 & 0.010 & 0.01 & 0.008 & 0.014 & 0.01 & 0.01 & 0.0145 \\
\hline $\mathrm{Fe}$ & 0.050 & 0.086 & 0.069 & 0.044 & 0.050 & 0.055 & 0.035 & 0.064 & 0.047 & 0.050 & 0.086 \\
\hline $\mathrm{Mo}$ & 0.016 & 0.018 & 0.019 & 0.018 & 0.016 & 0.022 & 0.019 & 0.019 & 0.016 & 0.020 & 0.025 \\
\hline $\mathrm{I}$ & 0.017 & 0.022 & 0.022 & 0.025 & 0.018 & 0.025 & 0.020 & 0.019 & 0.019 & 0.021 & 0.03 \\
\hline $\mathrm{Ba}$ & 0.033 & 0.034 & 0.045 & 0.043 & 0.038 & 0.049 & 0.042 & 0.040 & 0.039 & 0.040 & 0.059 \\
\hline $\mathrm{Mn}$ & 0.012 & 0.086 & 0.022 & 0.044 & 0.058 & 0.015 & 0.035 & 0.069 & 0.011 & 0.050 & 0.086 \\
\hline $\mathrm{Sr}$ & 0.093 & 0.012 & $\mathrm{bdL}$ & $\mathrm{bdL}$ & $\mathrm{bdL}$ & $\mathrm{bdL}$ & $\mathrm{bdL}$ & $\mathrm{bdL}$ & $\mathrm{bdL}$ & $\mathrm{bdL}$ & $\mathrm{bdL}$ \\
\hline
\end{tabular}


Table (7) Trace elements concentration for all tayps seeds of $\operatorname{grapes}(\boldsymbol{\mu g} / \mathrm{g})$

\begin{tabular}{|c|c|c|c|c|c|c|c|c|c|c|c|}
\hline Element & SZK- & SZK- & SZK- & SZK- & SZK- & SZK- & SZK- & SZK- & SZK- & SZKI- & SZK- \\
& 1 & 2 & 3 & 4 & 5 & 6 & 7 & 8 & 9 & 10 & 11 \\
\hline $\mathrm{Ti}$ & $\mathrm{bdL}$ & $\mathrm{bdL}$ & $\mathrm{bdL}$ & 7.20 & $\mathrm{bdL}$ & $\mathrm{bdL}$ & 8.3 & $\mathrm{bdL}$ & $\mathrm{bdL}$ & $\mathrm{bdL}$ & 10.4 \\
\hline $\mathrm{Cr}$ & 5.00 & 13.2 & 4.70 & 4.90 & 4.60 & 3.70 & 16.4 & 4.8 & 11.0 & 4.5 & 21.0 \\
\hline $\mathrm{CO}$ & 1.2 & 2.51 & 12.21 & 0.80 & 1.10 & 0.90 & 0.80 & 1.10 & 0.50 & 1.50 & 2.20 \\
\hline $\mathrm{Ni}$ & 1.3 & 2.40 & 10.6 & 1.90 & 0.70 & 1.50 & 8.10 & 1.50 & 0.30 & 1.60 & 4.10 \\
\hline $\mathrm{Cu}$ & 9.2 & 8.70 & 14.2 & $8.3 ` 1$ & 7.80 & 7.50 & 6.80 & 17.7 & 7.90 & 9.90 & 8.90 \\
\hline $\mathrm{Rb}$ & 1.80 & 1.01 & 1.50 & 1.70 & 8.41 & 1.7 & 9.39 & 10.9 & 1.8 & 1.9 & 1.8 \\
\hline $\mathrm{Ag}$ & 3.00 & 4.509 & 3.90 & 4.8 & 4.10 & 5.40 & 3.7 & 370 & 4.40 & 4.70 & 6.10 \\
\hline $\mathrm{Cd}$ & 3.90 & 4.40 & 4.40 & 4.70 & 4.40 & 6.10 & 4.40 & 4.80 & 4.40 & 5.30 & 7.80 \\
\hline $\mathrm{Sn}$ & 5.5 & 7.10 & 5.10 & 5.10 & 4.8 & 6.9 & 7.00 & 5.80 & 8.10 & 5.20 & 11 \\
\hline $\mathrm{Sb}$ & 6.00 & 7.70 & 6.8 & 7.70 & 7.50 & 8.7 & $7 . / 40$ & 5.80 & 7.80 & 8.20 & 10 \\
\hline $\mathrm{W}$ & 1.5 & 1.5 & 1.5 & 1.40 & 1.40 & 1.3 & 1.3 & 1.50 & 1.4 & 1.3 & 1.5 \\
\hline $\mathrm{Te}$ & 8.2 & 12.0 & 11.5 & 13.0 & 11.0 & 13.0 & 4.2 & 300 & 39 & 40 & 59 \\
\hline $\mathrm{Br}$ & 800 & 800 & 500 & 1000 & 500 & 500 & 700 & 1000 & 600 & 500 & 1800 \\
\hline $\mathrm{Pb}$ & $<600$ & 2400 & 300 & 1300 & 1600 & 800 & 3300 & 700 & 2500 & 3000 & 1000 \\
\hline $\mathrm{Th}$ & $<600$ & 1100 & 800 & 500 & 900 & 800 & 500 & 400 & 1200 & 1800 & 1000 \\
\hline $\mathrm{V}$ & $<0.7$ & 1.50 & 2.3 & 2.3 & 2.20 & 1.80 & 2.00 & 1.70 & 1.30 & 1.30 & 1.20 \\
\hline
\end{tabular}

\section{References:}

1. Asai A.A., 1987, The element in unani medicine and its Scientific Relevance, Elements in health and disease, WHO, IVE.

2. Williane E.j. and weather all $\mathrm{m} \mathrm{D}$, 1999, Abnormal, horgloblnes in Africa, axford.

3. Mahdi K.H. , Al kubaisy R.K., AL Mousawiy M.A.A, 2005, Analysis of different typical leaves by XRF technique ,Ibn Al- haitham of pureal a phy. Seic.18.(3).

4. A L- Incale B. R., Kuzgozu N.O., Transin H. and stocks R. , 2009 , Invernataval journal of food and sicnceened Nutrition , 60:32-39.

5. Nikdel S., Naay S. , 1985 , Scientific Resarch deverlprmants of citvis , 18:42-53.

6. Farhan S.R., ALjaberi S.M. , Itawi R.K., Jamal M. , Nassar M.A. , 2002, stady of elements content of Iraqi dosent by INNA and XRF technique J. Scie. Englanding,61(69).
7. Alsaidi E.H. ,2002, "Grapes Production)", Ministry of Higher Education and Scientific Research , College of Agriculture, University of Almousil .

8. Aljobori S.M., Alkabaisy R.K. , Mahdi H.K. , 2002,Detenments of major and trace elements in Iraqi vegetable samples by XRE, Iraqi J. Phys. and math. 1(3).

9. Abdulla R. , 1990, Detrertion of some element concentration in vegetable by using XRE method, Thsis, College of Education AbnAl-Hathim, Baghdad University.

10. Catterall F. , J.M. Souquet, V. Cheynier ,C. Santos-Buelga, M.N. Clifford and C. Ioannides ,2000, Differential Modulation of the Genotoxicity of food Carcinogens by Naturally Occurring Monomeric and diemeric polyphenolics, Enviromental and Molecular Mutagenesis 35 :86-98 . 


\section{تحليل فواكه العنب وبذورها باستعمال تقتية XRF}

** شاكرمحود الجبوري

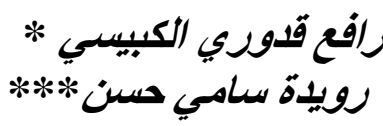

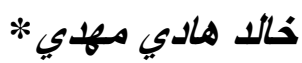

| ملية التربية - جامعة بغداد

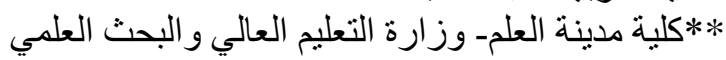

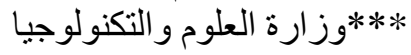

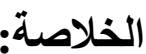

العنب وبذوره مهمة جدا في الدراسات البيئية و الصحية لـألا يهدف هذا البحث الى ايجاد نوعية وتر اكيز

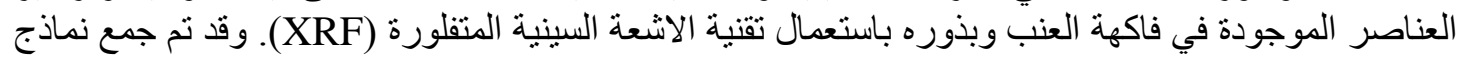

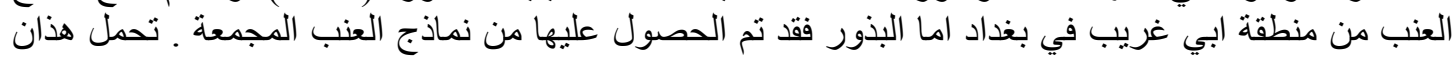

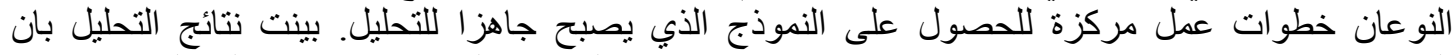

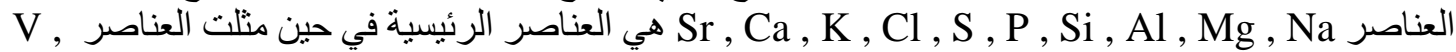
Te , Sp كعناصر ثنانوية وقد تراوحت تر اكيز العناصر البقية من Ba , I , Sr , Fe

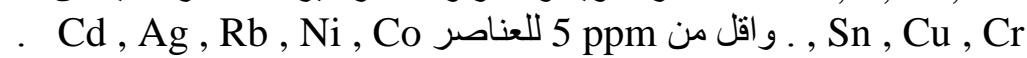

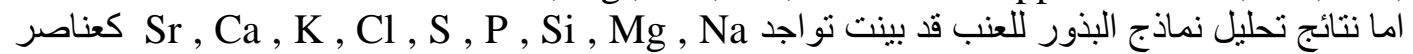

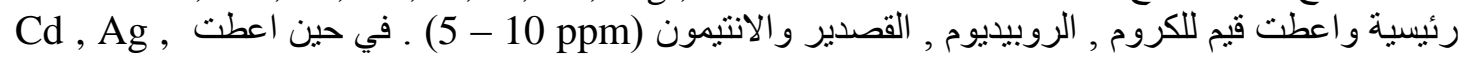
Rb , Ni , Co 